\title{
Erratum to: Scaled Entropy for Dynamical Systems
}

\author{
Yun Zhao' ${ }^{1}$. Yakov Pesin ${ }^{2}$
}

Published online: 29 January 2016

(C) Springer Science+Business Media New York 2016

\section{Erratum to: J Stat Phys (2015) 158:447-475 DOI 10.1007/s10955-014-1133-5}

In [4] using the Carathéodory approach as described in [3], we introduced the notions of scaled topological and scaled metric entropies and we described some of their basic properties. Unfortunately, one of the results we stated is not true. In addition, we did not mention an important work in the area by Katok and Thouvenot [1] in which they introduced the notion of slow metric entropy based on an approach that utilizes the Hamming metrics. While our scaled metric entropy may not coincide with the slow metric entropy and they have different properties, the relations between them are instructive and help better understand the rescaling phenomena in dynamics. This note is intended to cover the gap and to correct a mistake in our paper.

\section{Scaled Topological Entropy}

We begin by briefly recalling the definitions of scaled topological and scaled metric entropies. Consider a continuous transformation $T: X \rightarrow X$ of a compact metric space $X$ equipped with metric $d$. A sequence of positive numbers $\mathbf{a}=\{a(n)\}_{n \geq 1}$ is a scaled sequence if it is monotonically increasing to infinity.

The online version of the original article can be found under doi:10.1007/s10955-014-1133-5.

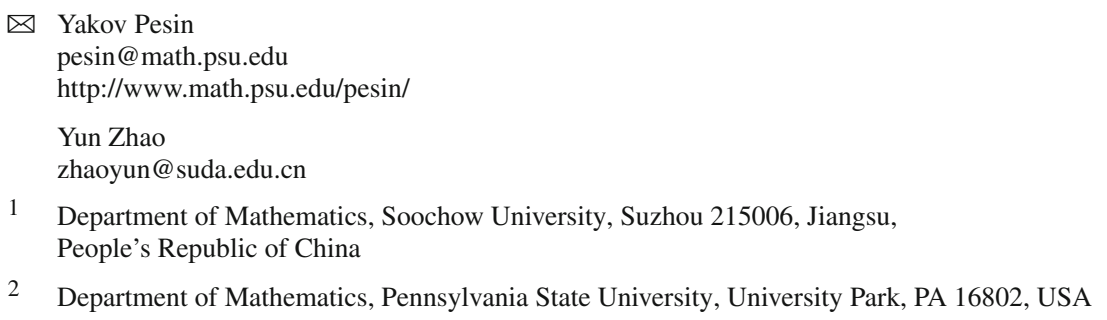


Given $\epsilon>0, n \in \mathbb{N}$ and $x \in X$, denote by $B_{n}(x, \epsilon)=\left\{y \in X: d_{n}(x, y)<\epsilon\right\}$ the Bowen's ball of radius $\epsilon$ centered at $x$ of length $n$, where $d_{n}(x, y):=\max \left\{d\left(T^{i}(x), T^{i}(y)\right): 0 \leq i<\right.$ $n\}$. Following the general Carathéodory construction of dimension-like characteristics (see [3]), given a scaled sequence $\mathbf{a}=\{a(n)\}$, for each subset $Z \subset X$ and each $\alpha, N>0$ set

$$
\begin{aligned}
M(Z, \alpha, N, \epsilon, \mathbf{a})=\inf & \left\{\sum_{i} \exp \left(-\alpha a\left(n_{i}\right)\right):\right. \\
& \left.\bigcup_{i} B_{n_{i}}\left(x_{i}, \epsilon\right) \supset Z, x_{i} \in X \text { and } n_{i} \geq N \text { for all } i\right\} .
\end{aligned}
$$

Since $M(Z, \alpha, N, \epsilon, \mathbf{a})$ is monotonically increasing with $N$,

$$
m(Z, \alpha, \epsilon, \mathbf{a})=\lim _{N \rightarrow \infty} M(Z, \alpha, N, \epsilon, \mathbf{a}) .
$$

We denote the jump-up point of $m(Z, \alpha, \epsilon, \mathbf{a})$ by

$$
E_{Z}(T, \mathbf{a}, \epsilon)=\inf \{\alpha: m(Z, \alpha, \epsilon, \mathbf{a})=0\}=\sup \{\alpha: m(Z, \alpha, \epsilon, \mathbf{a})=+\infty\} .
$$

The following limit

$$
E_{Z}(T, \mathbf{a})=\lim _{\epsilon \rightarrow 0} E_{Z}(T, \mathbf{a}, \epsilon)
$$

is called the scaled topological entropy of $T$ on the set $Z$.

For any subset $Z \subset X$, let $\aleph(Z, n, \epsilon)$ denote the smallest number of Bowen's balls $\left\{B_{n}(x, \epsilon)\right\}$ whose union covers the set $Z$. For any scaled sequence $\mathbf{a}=\{a(n)\}$, define

$$
\begin{aligned}
& \underline{E}_{Z}(T, \mathbf{a}, \epsilon)=\liminf _{n \rightarrow \infty} \frac{1}{a(n)} \log \aleph(Z, n, \epsilon), \\
& \bar{E}_{Z}(T, \mathbf{a}, \epsilon)=\limsup _{n \rightarrow \infty} \frac{1}{a(n)} \log \aleph(Z, n, \epsilon) .
\end{aligned}
$$

The quantities

$$
\underline{E}_{Z}(T, \mathbf{a}):=\lim _{\epsilon \rightarrow 0} \underline{E}_{Z}(T, \mathbf{a}, \epsilon), \quad \bar{E}_{Z}(T, \mathbf{a}):=\lim _{\epsilon \rightarrow 0} \bar{E}_{Z}(T, \mathbf{a}, \epsilon)
$$

are called lower and upper scaled topological entropies of $T$ on the set $Z$.

We stress that the set $Z$ in the definitions of scaled topological entropies need not be invariant nor compact. One can show that

$$
E_{Z}(T, \mathbf{a}) \leq \underline{E}_{Z}(T, \mathbf{a}) \leq \bar{E}_{Z}(T, \mathbf{a}) .
$$

Further, one can show that the scaled topological entropy as well as the lower and upper scaled topological entropies are invariant under a topological conjugacy.

\section{Scaled Topological Entropy: An Erratum}

In this section, we point out that Theorem 2.6 in [4] is wrong. This theorem claimed that scaled topological entropy and lower and upper scaled topological entropies of a set $Z$ coincide provided $Z$ is invariant and compact. In fact, Example 4.5 in [4] shows that there exists a non-degenerate dynamically coherent Bott system $\left(\mathcal{L}, \phi_{H}, f\right)$ such that

$$
E_{\mathcal{L}}\left(\phi_{H}^{1}, \mathbf{a}\right)<\bar{E}_{\mathcal{L}}\left(\phi_{H}^{1}, \mathbf{a}\right)
$$


where $\phi_{H}^{1}$ is the time one map of the Hamiltonian flow $\phi_{H}$ and $\mathbf{a}=\{\log n\}$. In this case, while the scaled sequence $\{\log n\}$ is not sub-additive it is equivalent to the scaled sequence $\{\log n+1\}$ which is sub-additive and hence the scaled topological entropies computed with respect to these two sequences must coincide. This shows that Theorem 2.6 is wrong.

\section{Scaled Metric Entropy}

Given a $T$-invariant measure $\mu$ and a scaled sequence $\mathbf{a}=\{a(n)\}$, let

$$
\begin{aligned}
E_{\mu}(T, \mathbf{a}, \epsilon) & =\inf \left\{E_{Z}(T, \mathbf{a}, \epsilon): \mu(Z)=1\right\} \\
& =\lim _{\delta \rightarrow 0} \inf \left\{E_{Z}(T, \mathbf{a}, \epsilon): \mu(Z) \geq 1-\delta\right\}
\end{aligned}
$$

and then let

$$
E_{\mu}(T, \mathbf{a})=\lim _{\epsilon \rightarrow 0} E_{\mu}(T, \mathbf{a}, \epsilon) .
$$

We call the quantity $E_{\mu}(T, \mathbf{a})$ the scaled metric entropy of $T$ with respect to $\mu$ (and the scaled sequence a). Let further

$$
\begin{aligned}
& \underline{E}_{\mu}(T, \mathbf{a}, \epsilon)=\lim _{\delta \rightarrow 0} \inf \left\{\underline{E}_{Z}(T, \mathbf{a}, \epsilon): \mu(Z) \geq 1-\delta\right\}, \\
& \bar{E}_{\mu}(T, \mathbf{a}, \epsilon)=\lim _{\delta \rightarrow 0} \inf \left\{\bar{E}_{Z}(T, \mathbf{a}, \epsilon): \mu(Z) \geq 1-\delta\right\} .
\end{aligned}
$$

We call the quantities

$$
\begin{aligned}
& \underline{E}_{\mu}(T, \mathbf{a})=\lim _{\epsilon \rightarrow 0} \underline{E}_{\mu}(T, \mathbf{a}, \epsilon), \\
& \bar{E}_{\mu}(T, \mathbf{a})=\lim _{\epsilon \rightarrow 0} \bar{E}_{\mu}(T, \mathbf{a}, \epsilon)
\end{aligned}
$$

respectively the lower and upper scaled metric entropy of $T$ with respect to $\mu$ (and the scaled sequence a). For any scaled sequence $\mathbf{a}=\{a(n)\}$ we can easily show that

$$
E_{\mu}(T, \mathbf{a}) \leq \underline{E}_{\mu}(T, \mathbf{a}) \leq \bar{E}_{\mu}(T, \mathbf{a}) .
$$

For a $T$-invariant ergodic measure $\mu$ and a scaled sequence $\mathbf{a}=\{a(n)\}$ satisfying $\lim _{n \rightarrow \infty} \frac{a(n+1)}{a(n)}=1$, we define the lower and upper scaled Brin-Katok's local entropies as follows:

$$
\begin{aligned}
& \underline{h}_{\mu}(T, \mathbf{a}):=\lim _{\epsilon \rightarrow 0} \liminf _{n \rightarrow \infty}-\frac{1}{a(n)} \log \mu\left(B_{n}(x, \epsilon)\right), \\
& \bar{h}_{\mu}(T, \mathbf{a}):=\lim _{\epsilon \rightarrow 0} \limsup _{n \rightarrow \infty}-\frac{1}{a(n)} \log \mu\left(B_{n}(x, \epsilon)\right) .
\end{aligned}
$$

The following theorem gives the relations between various versions of scaled metric entropies (see Theorem 3.3 in [4]).

Theorem 3.1 Let $\mathbf{a}=\{a(n)\}$ be a scaled sequence satisfying $\lim _{n \rightarrow \infty} \frac{a(n+1)}{a(n)}=1$. For any $T$-invariant ergodic measure $\mu$ we have

$$
\underline{h}_{\mu}(T, \mathbf{a}) \leq E_{\mu}(T, \mathbf{a}) \leq \underline{E}_{\mu}(T, \mathbf{a}) \leq \bar{E}_{\mu}(T, \mathbf{a}) \leq \bar{h}_{\mu}(T, \mathbf{a}) .
$$

One can show that the scaled metric entropy as well as the lower and upper scaled metric entropies are invariant under a topological conjugacy. 


\section{Slow Metric Entropy}

We introduce the notion of slow metric entropy following the work of Katok and Thouvenot [1]. In this paper they used the slow entropy as a technical tool to obtain smooth realizations of commuting measure preserving transformations. However, for our purposes we will restrict ourself to $\mathbb{Z}$-actions.

Let $\xi=\left\{C_{1}, \ldots, C_{p}\right\}$ be a finite measurable partition of $X$. For a point $x$ in $X$, the $\xi$-name of $x$ is the sequence $\left\{\xi_{i}(x)\right\}_{i \geq 0}$ where $\xi_{i}(x)=l$ if $T^{i}(x) \in C_{l}$ for some $1 \leq l \leq p$. Denote by $\xi_{[0, n)}(x)$ the sequence $\xi_{0}(x), \ldots, \xi_{n-1}(x)$. Given $x, y \in X$, the Hamming distance $d_{H}$ between $\xi_{[0, n)}(x)$ and $\xi_{[0, n)}(y)$ is defined by

$$
d_{H}\left(\xi_{[0, n)}(x), \xi_{[0, n)}(y)\right)=\frac{1}{n} \sharp\left\{0 \leq i<n: \xi_{i}(x) \neq \xi_{i}(y)\right\} .
$$

Given $x \in X$ and $\epsilon>0$, the ball $B_{H}(x, \epsilon)$ centered at $x$ of radius $\epsilon$ in the Hamming distance is defined as

$$
B_{H}(x, \epsilon)=\left\{y \in X: d_{H}\left(\xi_{[0, n)}(x), \xi_{[0, n)}(y)\right)<\epsilon\right\}
$$

For any $T$-invariant measure $\mu, 0<\delta<1$ and $n>0$ denote by $S_{d_{H}, \mu}^{\xi}(\epsilon, \delta, n)$ the minimal number of balls of radius $\epsilon$ in the Hamming distance whose union has measure $\geq 1-\delta$. Given a scaled sequence $\mathbf{a}=\{a(n)\}$, define

$$
\bar{S}_{\mu}(\xi, \epsilon, \delta):=\limsup _{n \rightarrow \infty} \frac{1}{a(n)} \log S_{d_{H}, \mu}^{\xi}(\epsilon, \delta, n) .
$$

Let further

$$
\bar{S}_{\mu}(\xi):=\lim _{\epsilon \rightarrow 0} \lim _{\delta \rightarrow 0} \bar{S}_{\mu}(\xi, \epsilon, \delta) .
$$

The quantity

$$
\bar{S}(\mu):=\sup _{\xi} \bar{S}_{\mu}(\xi)
$$

where the supremum is taken over all finite partitions of $X$, is called the upper slow metric entropy of $T$. Similarly, if lim sup in (4.1) is replaced by lim inf, one gets the quantities $\underline{S}_{\mu}(\xi, \epsilon, \delta)$ and $\underline{S}_{\mu}(\xi)$. The lower slow metric entropy of $T$ is defined as

$$
\underline{S}(\mu):=\sup _{\xi} \underline{S}_{\mu}(\xi) .
$$

We stress that the scaled sequences above should grow to infinity slower than the standard sequence $a(n)=n$, hence, the name slow entropy. A crucial property of lower and upper slow metric entropies is that they are invariants under an isomorphism of measurable spaces (see [1] for details).

The following theorem describes some relations between lower (upper) scaled metric entropy and lower (upper) slow metric entropy.

Theorem 4.1 Let $\mathbf{a}=\{a(n)\}$ be a scaled sequence, for any $T$-invariant ergodic measure $\mu$ we have

$$
\bar{S}(\mu) \leq \bar{E}_{\mu}(T, \mathbf{a}) \text { and } \underline{S}(\mu) \leq \underline{E}_{\mu}(T, \mathbf{a}) .
$$


Proof We present the proof of the first inequality as the other one can be proven in a similar fashion.

Fix a small number $\beta>0$ and choose a finite partition $\xi=\left\{C_{1}, \ldots, C_{p}\right\}$ of $X$ such that $\mu(\partial \xi)=0$ and $\bar{S}_{\mu}(\xi) \geq \bar{S}(\mu)-\beta$, where $\partial \xi=\bigcup_{A \in \xi} \partial A$ and $\partial A$ denotes the boundary of the set $A$.

For $\epsilon>0$ let

$$
U_{\epsilon}(\xi)=\{x \in X: \text { the ball } B(x, \epsilon) \text { is not contained in } \xi(x)\}
$$

where $\xi(x)$ is the element of the partition $\xi$ which contains the point $x$. Since $\bigcap_{\epsilon>0} U_{\epsilon}(\xi)=$ $\partial \xi$ we obtain that $\mu\left(U_{\epsilon}(\xi)\right) \rightarrow 0$ as $\epsilon \rightarrow 0$. Therefore, for any $\alpha>0$ one can choose $\epsilon_{0}>0$ such that $\mu\left(U_{\epsilon}(\xi)\right)<\alpha$ for any $0<\epsilon \leq \epsilon_{0}$. For each positive integer $N$, let

$$
A_{N}=\left\{x \in X: \frac{1}{n} \sum_{i=0}^{n-1} \chi_{U_{\epsilon}(\xi)}\left(T^{i}(x)\right)<\alpha \text { for any } n \geq N\right\} .
$$

It follows from the Birkhoff ergodic theorem that the sets $A_{N}$ are nested and exhaust $X$ up to a set of measure zero. For a small number $\delta>0$, pick $N_{0}$ such that $\mu\left(A_{N}\right) \geq 1-\delta$ for any $N \geq N_{0}$. In the following we fix such a positive integer $N$.

Given a point $x \in X$ and any $n \geq N$, if $y \in B_{n}(x, \epsilon)$ then for any $0 \leq i \leq n-1$ either $T^{i}(x)$ and $T^{i}(y)$ belong to the same element of the partition $\xi$ or $T^{i}(x) \in U_{\epsilon}(\xi)$. Hence, if $x \in A_{N}$ and $y \in B_{n}(x, \epsilon)$, then

$$
d_{H}\left(\xi_{[0, n)}(x), \xi_{[0, n)}(y)\right)<\frac{n \alpha}{n}=\alpha .
$$

It follows that

$$
S_{d_{H}, \mu}^{\xi}(\alpha, \delta, n) \leq \aleph\left(A_{N}, n, \epsilon\right) .
$$

To finish the proof of the theorem, for any subset $Z \subset X$ with $\mu(Z) \geq 1-\delta$ let us consider the set $\widetilde{Z}=Z \cap A_{N}$. Clearly, $\mu(\widetilde{Z}) \geq 1-2 \delta$. It yields that

$$
S_{d_{H}, \mu}^{\xi}(\alpha, 2 \delta, n) \leq \aleph(\widetilde{Z}, n, \epsilon) \leq \aleph(Z, n, \epsilon) .
$$

Hence, for any scaled sequence $\mathbf{a}=\{a(n)\}$ we have

$$
\bar{S}_{\mu}(\xi, \alpha, 2 \delta)=\limsup _{n \rightarrow \infty} \frac{1}{a(n)} \log S_{d_{H}, \mu}^{\xi}(\alpha, 2 \delta, n) \leq \bar{E}_{Z}(T, \mathbf{a}, \epsilon) .
$$

Since $Z$ is arbitrary, we find that

$$
\bar{S}_{\mu}(\xi, \alpha, 2 \delta) \leq \inf \left\{\bar{E}_{Z}(T, \mathbf{a}, \epsilon): \mu(Z) \geq 1-\delta\right\} .
$$

Letting $\delta \rightarrow 0$, we have that

$$
\lim _{\delta \rightarrow 0} \bar{S}_{\mu}(\xi, \alpha, 2 \delta) \leq \bar{E}_{\mu}(T, \mathbf{a}, \epsilon)
$$

Letting $\alpha \rightarrow 0$ in the above inequality and taking into account that this implies that $\epsilon \rightarrow 0$ we obtain that

$$
\bar{S}(\mu)-\beta<\bar{S}_{\mu}(\xi) \leq \bar{E}_{\mu}(T, \mathbf{a}) .
$$

Since $\beta$ is arbitrary this implies the desired result. 


\section{An Example}

In this section, we analyze the Example 4.7 in [4] to illustrate that: (1) the inequality in Theorem 3.1 can be strict and (2) the scaled metric entropy may not be invariant under an isomorphism of measurable spaces.

Let $T$ be a rotation by an irrational number $\theta$ on $[0,1)$. Since $T$ is an isometry, by Example 4.6 in [4] we know that the system $(X, T)$ has zero upper scaled topological entropy with respect to any scaled sequence. For any scaled sequence $\mathbf{a}=\{a(n)\}$ and $T$-invariant ergodic measure $\mu$ it follows from Theorem 4.1 that

$$
0 \leq \underline{S}(\mu) \leq \bar{S}(\mu) \leq \bar{E}_{\mu}(T, \mathbf{a}) \leq \bar{E}_{X}(T, \mathbf{a})=0 .
$$

The above facts together with (3.1) imply that for any scaled sequence $\mathbf{a}=\{a(n)\}$ and any $T$-invariant ergodic measure $\mu$ we have

$$
\underline{S}(\mu)=\bar{S}(\mu)=E_{\mu}(T, \mathbf{a})=\underline{E}_{\mu}(T, \mathbf{a})=\bar{E}_{\mu}(T, \mathbf{a})=0 .
$$

Let $\mu$ be the Lebesgue measure on $[0,1)$. Denote by $\mathcal{P}_{n}$ the partition of $[0,1)$ generated by the orbit $\{-k \theta\}, 0 \leq k \leq n$ that is $\mathcal{P}_{n}=\bigvee_{i=0}^{n-1} T^{-i} \mathcal{P}$, where the partition $\mathcal{P}=\{[0,1-$ $\theta),[1-\theta, 1)\}$. Further, for $x \in[0,1)$, we denote by $\mathcal{P}_{n}(x)$ the element of the partition $\mathcal{P}_{n}$ that contains $x$. For an irrational number $\theta \in(0,1)$, set

$$
\eta:=\sup \left\{t>0: \liminf _{j \rightarrow \infty} j^{t}\|j \theta\|=0\right\},
$$

where $\|j \theta\|$ denotes the distance to the nearest integer.

Now consider the full shift $\sigma$ on the one-sided symbolic space $\Sigma_{2}^{+}$and the map $\phi$ : $\Sigma_{2}^{+} \rightarrow[0,1)$ given by $\phi(\omega)=\bigcap_{i=0}^{\infty} T^{-i} P_{\omega_{i}}$. This map determines a symbolic extension of the irrational rotation, where $\omega_{i}$ is the $i$ th symbol of the word $\omega$ and $P_{0}=[0,1-\theta), P_{1}=$ $[1-\theta, 1)$. Let $\xi$ be the natural partition of $\left(\Sigma_{2}^{+}, \sigma\right)$ into 1-cylinders, i.e., $\xi=\left\{A_{0}, A_{1}\right\}$ with $A_{0}=\left\{\omega \in \Sigma_{2}^{+}: \omega_{0}=0\right\}$ and $A_{1}=\left\{\omega \in \Sigma_{2}^{+}: \omega_{0}=1\right\}$. The map $\phi$ maps the partition $\xi$ onto $\mathcal{P}$. Moreover, we obtain an ergodic shift invariant measure $m$ on $\Sigma_{2}^{+}$such that $\phi_{*} m=\mu$. Furthermore, $\left(\Sigma_{2}^{+}, \mathcal{B}_{1}, m, \sigma\right)$ is isomorphic via $\phi$ to $\left([0,1), \mathcal{B}_{2}, \mu, T\right)$, where $\mathcal{B}_{1}$ and $\mathcal{B}_{2}$ are sigma algebras on $\Sigma_{2}^{+}$and $[0,1)$ generated by the partitions $\xi$ and $\mathcal{P}$ respectively.

We denote by $C_{\xi_{n}}(\omega)$ the element of the refined partition $\bigvee_{i=0}^{n-1} \sigma^{-1} \xi$ which contains the point $\omega$. Since $\eta=1$ for almost every $\theta$, we conclude that by (i) of Theorem 1.1 in [2],

$$
\lim _{n \rightarrow \infty}-\frac{\log m\left(C_{\xi_{n}}(\omega)\right)}{\log n}=1
$$

for $m$-almost every $\omega$. Let $\mathbf{a}=\{\log n\}$, by Theorem 3.1 we have

$$
\underline{h}_{m}(\sigma, \mathbf{a})=E_{m}(\sigma, \mathbf{a})=\underline{E}_{m}(\sigma, \mathbf{a})=\bar{E}_{m}(\sigma, \mathbf{a})=\bar{h}_{m}(\sigma, \mathbf{a})=1 .
$$

Compare to (5.1), this implies the scaled metric entropy and lower and upper scaled metric entropies are not invariant under a metric isomorphism. On the other hand, since $\underline{S}(\mu)$ and $\bar{S}(\mu)$ are isomorphism invariant, by (5.1) we have

$$
\underline{S}(m)=\underline{S}(\mu)=0 \text { and } \bar{S}(m)=\bar{S}(\mu)=0 .
$$

Hence, for the scaled sequence $\mathbf{a}=\{\log n\}$ we have

$$
\underline{S}(m)<\underline{E}_{m}(\sigma, \mathbf{a}) \text { and } \bar{S}(m)<\bar{E}_{m}(\sigma, \mathbf{a}) .
$$


Acknowledgments We would like to thank A. Katok for pointing out the mistake and for bringing our attention to his work with J.-P. Thouvenot. The first author was partially supported by NSFC 11371271 . The second author was partially supported by NSF grants 1101165 .

\section{References}

1. Katok, A., Thouvenot, J.-P.: Slow entropy type invariants and smooth realization of commuting measurepreserving transformations. Ann. Inst. H. Poincare Probab. Stat. 33(3), 323-338 (1997)

2. Kim, D., Park, K.: The first return time properties of an irrational rotation. Proc. Am. Math. Soc. 136(11), 3941-3951 (2008)

3. Pesin, Y.: Dimension Theory in Dynamical Systems: Contemporary Views and Applications. University of Chicago Press, Chicago (1997)

4. Zhao, Y., Pesin, Y.: Scaled entropy for dynamical systems. J. Stat. Phys. 158, 447-475 (2015) 